\title{
RECUPERAÇÃO DO FÓSFORO RESIDUAL DO SOLO, DERIVADO DE UM TERMOFOSFATO MAGNESIANO COM DIFERENTES GRANULOMETRIAS E DO SUPERFOSFATO SIMPLES GRANULADO'
}

\author{
R. STEFANUTTR; E. MALAVOLTA²; T. MURAOKA² \\ ${ }^{2}$ Centro de Energia Nuclear na Agricultura - CENAJSP, C.P. 96, CEP: 13400-970 - Piracicaba,SP.
}

RESUMO: $O$ trabalho compara a recuperação do $\mathrm{P}_{2} \mathrm{O}_{5}$ residual através de cultivos sucessivos em um solo que recebeu doses crescentes de um termofosfato magnesiano com diferentes granulometrias e superfosfato simples granulado. Os resultados obtidos pela determinaçăo do $P$ extraido pelas plantas em 7 cultivos em vaso permitem concluir que o termofosfato na forma pó, apresenta efeito residual semelhante ao do superfosfato simples granulado; a granulometria mais grosseira do produto termofosfato resultou em menor recuperação do fósforo residual.

Descritores: termofosfato magnesiano, fósforo, fósforo residual, granulometria, fontes de fósforo

\section{RECUPERATION OF RESIDUAL PHOSPHORUS FROM MAGNESIUM THERMOPHOSPHATE OF DIFFERENT GRANULE SIZES AND FROM GRANULATED ORDINARY SUPERPHOSPHATE}

ABSTRACT: This paper compares the recovery of the residual $\mathrm{P}_{2} \mathrm{O}_{5}$ from magnesium thermophosphate (MPT) with different granule size, and from granular ordinary superphosphate(OSP). Seven successive crops were grown in pots in a greenhouse and their phosphorus content was analysed. Results demonstrated that powder MTP has the same residual effect as OSP. Coarse MTP, however, caused lower recovery of residual phosphorus.

Key Words: Magnesium thermophosphate, phosphoras, residual phosphorus, particles size, ordinary superphosphate

\section{INTRODUÇĀO}

Os teores de fósforo na solução do solo, além de muito baixos, são insuficientes para suprir as necessidades de uma cultura. Particularmente, este nutriente está envolvido em processos de fixação, que podem ser permanentes para a maioria dos solos tropicais ácidos.

Aplicam-se quantidades muito maiores de $\mathbf{P}$ no solo do que as plantas retiram (MALAVOLTA, 1981; RAIJ et al., 1982). Outro fator que interfere é o aproveitamento inicial do fósforo aplicado no primeiro ano, que é muito baixo, da ordem de 5 a $20 \%$ para a maioria das culturas (MALAVOLTA \& KLIEMANN, 1985). Entretanto, o fósforo residual pode ser aproveitado pelas culturas nos anos seguintes, embora parte passe para formas menos disponiveis.

Os adubos fosfatados solúveis, como o superfosfato simples, são aplicados aos solos na forma de grânulos e, ao entrarem em contacto com o solo, ocorre uma rápida absorção de umidade, ocasionando a dissolução do $\mathbf{P}$ (VOLKWEISS \& RAIJ, 1977). No caso dos termofosfatos, o processo de dissolução do fósforo é mais lento, pois depende de reações químicas com o solo. Entretanto, com o tempo, o fósforo residual poderá se equiparar ou mesmo até superar os resultados da fonte solúvel em água.

Algumas propriedades fisicas dos adubos, como o tamanho das partículas, determinam maior ou menor aproveitamento do nutriente pelas plantas. No caso do fósforo solúvel, a granulação adequada do fertilizante e a correção da acidez do solo reduzem a fixação do elemento.

TERMAN et al. (1970) comentaram que a eficiência inicial de um fertilizante fosfatado é altamente afetada pela solubilidade e pelo tamanho do grânulo.

NOVOA \& NUNEZ (1974) analisaram os fertilizantes fosfatados solúveis e constataram que se comportam de maneira mais eficiente na forma de grânulos do que em pó.

\footnotetext{
${ }^{1}$ Parte da dissertação de mestrado do primeiro autor apresentada ao CENA/USP, Piracicaba, SP.
}

Sci. agric., Piracicaba, 52(2):233-238, mai./ago. 1995 
O trabalho visa avaliar a recuperação do $P$ residual de um termofosfato magnesiano com diferentes granulometrias, comparando-o com o superfosfato simples granulado.

\section{MATERIAL E METODOS}

O ensaio foi conduzido em vasos de polietileno com $2 \mathrm{~kg}$ de terra, em casa de vegetação do Centro de Energia Nuclear na Agricultura/CENA, Piracicaba. Foram empregadas amostras de um Latossolo Vermelho-Amarelo fase cerrado, textura média argilosa, apresentado o seguinte resultado da análise química: $\mathrm{pH}$ (água) $=4,6 ; \% \mathrm{C}=1,1 ; \mathrm{P}$ (Mehlich) $=1 \mathrm{ppm} ; \mathrm{K}=0,08 \mathrm{meq} / 100 \mathrm{ml} ; \mathrm{Ca}=0,4$ meq $/ 100 \mathrm{ml} ; \mathrm{H}+\mathrm{Al}=3,4 \mathrm{meq} / 100 \mathrm{ml} ; \mathrm{Mg}=0,3$ $\mathrm{meq} / 100 \mathrm{ml} ; \mathrm{S}-\mathrm{SO} 4=10 \mathrm{ppm} ; \mathrm{SB}=1,0 \mathrm{meq} / 100$ $\mathrm{ml} ; \mathrm{T}=4,5 \mathrm{meq} / 100 \mathrm{ml} ; \mathrm{V}=22 \%$, (RAIJ et al., 1987).

O solo foi corrigido elevando-se a saturação de bases para $60 \%$. Foram aplicadas doses crescentes $(0,50,100$ e $200 \mathrm{ppm}$ de $P)$ de superfosfato simples granulado e termofosfato magnesiano nas formas pó, grosso e granulado.

As características químicas e fisicas das fontes de fósforo utilizadas são apresentadas na TABELA1.

TABELA 1 - Características químicas e fisicas das fontes de fósforo

\begin{tabular}{|c|c|c|c|c|c|c|c|}
\hline Produtos & $\begin{array}{l}\text { Natureza } \\
\text { Física }\end{array}$ & $\begin{array}{c}\text { Passante } \\
\text { Peneira } \\
(\mathrm{mm})\end{array}$ & Total & $\begin{array}{l}\mathrm{O}_{3} \\
\text { Solúvel }\end{array}$ & $\mathrm{Ca}$ & $\mathbf{M g}$ & $\mathbf{S}$ \\
\hline MG Yoorin & Granular & 50 a 0,84 & 18,0 & $16,5^{*}$ & 17 & 7 & 0 \\
\hline MG Yoorin & Pó & 75 a 0,15 & 18,0 & $16,5^{*}$ & 20 & 9 & 0 \\
\hline MG Yoorin & Grosso & 100 a 0,84 & 18,0 & $11,0^{*}$ & 20 & 9 & 0 \\
\hline SPS & Granular & 100 a $1-2$ & 19,0 & 18,0 & 20 & 0 & 12 \\
\hline
\end{tabular}

*Solúvel em ácido cítrico - $2 \%$ SPS-Superfosfato Simples.

Foram cultivados em sucessão feijão, milho e trigo até o "esgotamento" do fósforo. Foi determinada a produção de matéria seca e o fósforo absorvido de cada cultivo, segundo MALAVOLTA et al. (1989).

O fósforo recuperado nos sete cultivos foi determinado algebricamente a partir do fósforo adicionado ao solo em cada tratamento, subtraindo-se o P extraído pelas plantas em cada cultivo.

\section{RESULTADOS E DISCUSSÃo}

As comparações com o $\mathbf{P}$ absorvido são fundamentadas no fato de que a absorção do elemento pela planta e seu crescimento, determinado pela produção de matéria seca, estão relacionadas com as quantidades disponiveis do elemento no solo. Esta inferência está de acordo com VOLKWEISS \& RAIJ (1977), que consideram que as raízes absorvem o $\mathbf{P}$ da solução do solo em quantidades proporcionais à sua concentração.

Sci. agric., Piracicaba, 52(2):233-238, mai./ago. 1995
O fósforo total absorvido nos sete cultivos e o fósforo total recuperado são apresentados na TABELA 2.

Os resultados evidenciam que o fósforo residual apresentou-se insuficiente para suprir adequadamente as plantas no VII cultivo, em todos os niveis e fontes, não havendo diferença significativa entre as fontes estudadas, neste cultivo. A partir do VI cultivo, o fósforo residual da fonte solúvel demonstrou sinais de esgotamento e ou de baixa disponibilidade para o suprimento das plantas em todos os niveis (TABELA 3).

Estes resultados indicam que o fósforo remanescente da adiçăo inicial não estava mais disponivel para as plantas. Uma das causas que contribui para explicar este fato é o processo de fixação do fósforo desenvolvido pelo solo e certamente intensificado com a acidificação que as terras dos vasos sofreram durante os cultivos (MALAVOLTA, 1981).

O Yoorin pó não apresentou diferenças no fornecimento de fósforo ao longo dos cultivos de 
TABELA 2 - Fósforo total absorvido nos sete cultivos após aplicação de 50,100 e 200 ppm de Yoorin nas formas pó, grosso e granulado, e de superfosfato simples; fósforo total recuperado.

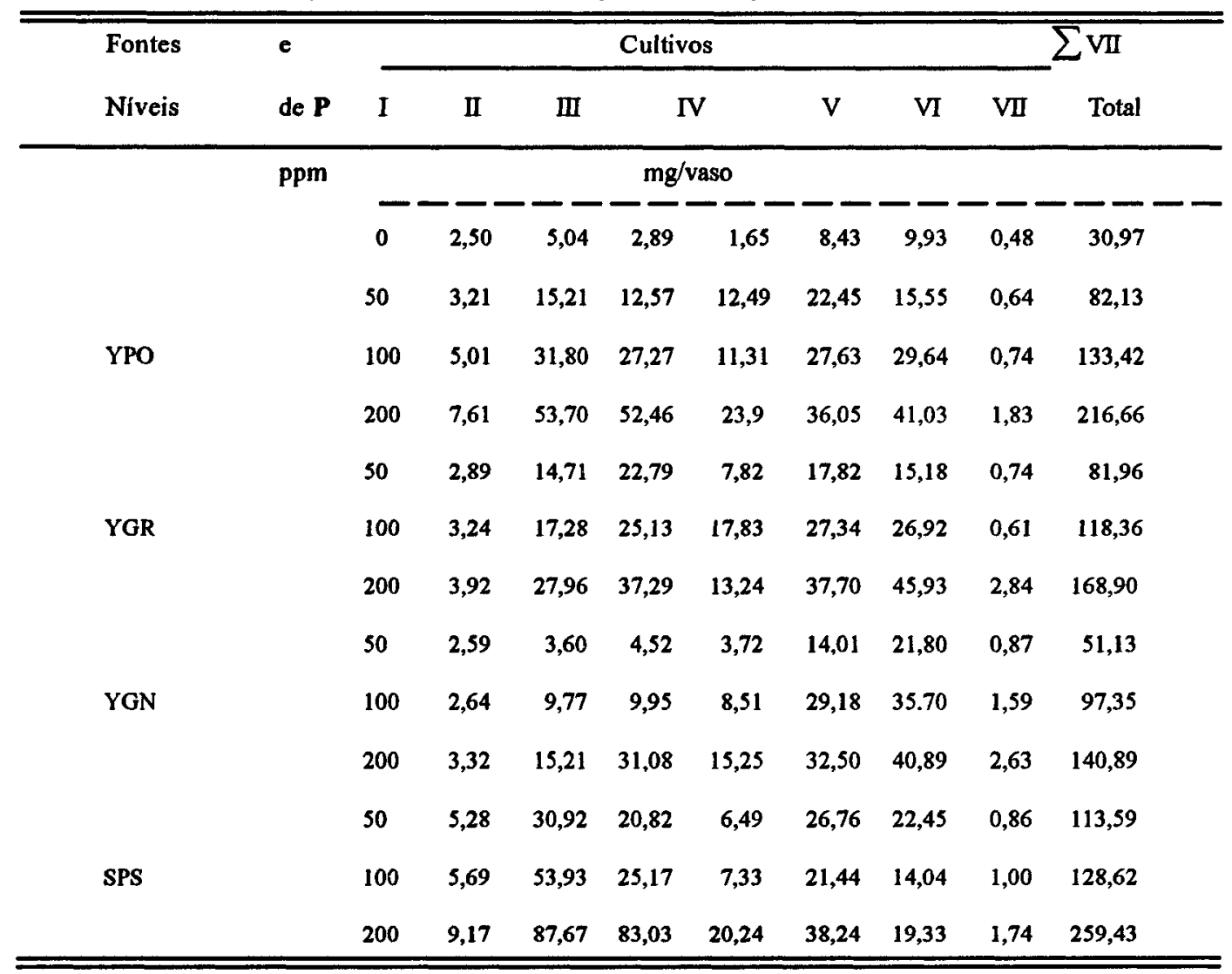

Cultivos: I-feijão; II, III, V e VI-milho; IV e VII trigo. YPO- Yoorin Pó; YGR- Yoorin Grosso; YGN- Yoorin Granulado; SPS-superfosfato simples.

milho. Foi a fonte mais regular no formecimento do nutriente para as plantas de milho, indicando que houve uma reposição constante do fósforo na solução do solo, até o VI cultivo.

A fonte Yoorin granulado foi inferior às demais fontes e comportou-se de modo oposto à fonte solúvel no processo de liberação de $\mathbf{P}$ ao longo dos cultivos, como pode ser observado na TABELA 3.

Na Figura 1 é destacado o fósforo recuperado em todos os cultivos. O maior destaque na recuperação de fósforo ocorre para a fonte superfosfato simples que, a partir do VI cultivo, no nível $50 \mathrm{ppm}$, ultrapassou a quantidade de fósforo adicionada. Esta é a situação desejável, porque indica que não ocorreu fixaçăo do fosfato aplicado.

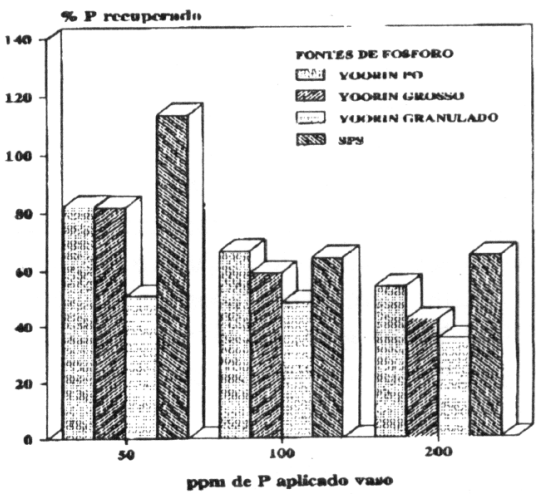

Figura 1- Fósforo (\%) extraído nos sete cultivos em função de diferentes fontes, granulometrias e níveis . 
TABELA 3- P recuperado nos diversos cultivos sob diferentes fontes de fósforo.

\begin{tabular}{|c|c|c|c|c|c|c|}
\hline \multirow[b]{2}{*}{ FONTES } & \multicolumn{6}{|c|}{ CULTIVOS } \\
\hline & II & III & $\overline{\mathrm{IV}}$ & $\mathrm{v}$ & VI & VII \\
\hline SPS & $44,39 \mathrm{aA}$ & $32,98 \mathrm{bA}$ & $8,92 \mathrm{eAB}$ & $23,73 \mathrm{cA}$ & $16,44 \mathrm{~dB}$ & $1,02 \mathrm{fAB}$ \\
\hline YOO PÓ & $26,44 \mathrm{aB}$ & $23,80 \mathrm{aB}$ & $12,35 \mathrm{bA}$ & $23,65 \mathrm{aA}$ & $24,04 \mathrm{aAB}$ & $0,92 \mathrm{cB}$ \\
\hline GROSSO & $16,25 \mathrm{bcc}$ & $22,02 \mathrm{abB}$ & $10,13 \mathrm{cAB}$ & $22,83 \mathrm{aA}$ & $24,49 \mathrm{aA}$ & $1,39 \mathrm{dAB}$ \\
\hline $\begin{array}{l}\text { GRANULA } \\
\text { DO }\end{array}$ & $8,11 \mathrm{cD}$ & $12,11 \mathrm{abc}$ & $7,26 \mathrm{cB}$ & $21,04 \mathrm{abA}$ & $27,08 \mathrm{aA}$ & $1,44 \mathrm{dA}$ \\
\hline DMS 5\% & 6,45 & 2,20 & 3,71 & 6,68 & 7,93 & 0,51 \\
\hline
\end{tabular}

Médias seguidas de letras minúsculas iguais, nas linhas, não diferem entre si a $5 \%$ e as seguidas de letras maiúsculas iguais, nas colunas, não diferem entre si a 5\% II- millho; III- milho; IV- trigo; V- milho; VI- milho; VII- milho. YPO-Yoorin Pó; YGR-Yoorin Grosso; YGN-Yoorin Granulado; SPS-superfosfato simples.

O TABELA 4 apresenta o fósforo residual calculado, existente no solo, à partir da dose inicial aplicada.
Embora alguns tratamentos ainda apresentassem fösforo remanescente, "contábil", em quantidade elevada (TABELA 4), a análise de solo pelo método da

TABELA 4 - Fósforo residual calculado para cada cultivo.

\begin{tabular}{|c|c|c|c|c|c|c|c|c|}
\hline \multirow{3}{*}{ Fonte } & \multirow{3}{*}{$\begin{array}{l}\text { Dose* } \\
\text { (mg) }\end{array}$} & \multicolumn{7}{|c|}{ Cultivos } \\
\hline & & \multicolumn{6}{|c|}{ (mg/vaso) } & \\
\hline & & I & II & III & IV & V & VI & VII \\
\hline Testemunha & 0 & $-2,49$ & $-7,5$ & $-10,42$ & $-12,08$ & $-20,56$ & $-30,49$ & $-30,97$ \\
\hline YP1 & 100 & 96,79 & 81,57 & 68,99 & 56,50 & 34,05 & 18,50 & 17,86 \\
\hline YP2 & 200 & 194,99 & 163,18 & 135,90 & 124,59 & 96,96 & 67,32 & 66,57 \\
\hline YP3 & 400 & 392,39 & 338,68 & 286,22 & 262,25 & 226,20 & 185,16 & 183,33 \\
\hline YGrl & 100 & 97,11 & 82,39 & 59,60 & 51,78 & 33,96 & 18,78 & 18,03 \\
\hline YGr2 & 200 & 196,76 & 179,49 & 154,35 & 136,52 & 109,18 & 82,25 & 81,64 \\
\hline YGr3 & 400 & 396,08 & 368,11 & 330,82 & 317,57 & 279,87 & 233,94 & 231,09 \\
\hline YGn1 & 100 & 97,41 & - 93,80 & 89,27 & 85,55 & 71,53 & 49,73 & 48,86 \\
\hline YGn2 & 200 & 197,36 & 187,59 & 177,64 & 169,12 & 139,94 & 104,23 & 102,64 \\
\hline YGn3 & 400 & 396,68 & 381,47 & 350,38 & 335,13 & 302,63 & 261,74 & 259,10 \\
\hline SPS1 & 100 & 94,72 & 63,79 & 42,97 & 36,48 & 9,72 & $-12,73$ & $-13,59$ \\
\hline SPS2 & 200 & 194,31 & 140,37 & 115,19 & 107,86 & 86,42 & 72,37 & 71,37 \\
\hline SPS3 & 400 & 390,83 & 303,15 & 220,12 & 199,88 & 161,64 & 142,30 & 140,56 \\
\hline
\end{tabular}

* fósforo aplicado antes do $\mathrm{I}^{\circ}$ Cultivo (mg/vaso); YP1- Yoorin pó 50 ppm; YP2- Yoorim pó 100 ppm; YP3Yoorim pó 200 ppm; YGrl- Yoorin grosso 50 ppm; YG2- Yoorin grosso 100 ppm; YGr3- Yoorin grosso 200 ppm; YGn1-Yoorin granulado 50 ppm; YGn2- Yoorin granulado $100 \mathrm{ppm}$; YGn3-Yoorin granulado $200 \mathrm{ppm}$; SPS1- Superfosfato Simples 50 ppm; SPS2-Superfosfato Simples 100 ppm; SPS3- Superfosfato Simples 200 ppm

resina (TABELA 5) mostra o baixo teor de $P$ disponivel. STEFANUTTI et al. (1992) compararam métodos de extração de $P$ e concluíram que a resina apresentou resultados mais representativos da extração de P e produção de matéria seca ao longo dos cultivos.
Os resultados das análises de solo após o VII cultivo, confirmam a acidificação do solo e o baixo teor de $\mathbf{P}$ disponível pelo método da resina (TABELA 5). Pelo teste de Tukey aplicado às médias do fósforo extraído e acumulado para as diferentes fontes, verificam-se diferenças significativas entre es- 
RECUPERAÇÃO DO FÓSFORO RESIDUAL DO SOLO ...

TABELA 5 - A análise do solo após o VII- Cultivo. Método da resina e $\mathrm{pH} \mathrm{em} \mathrm{CaCl}_{2}$.

\begin{tabular}{|c|c|c|c|c|c|c|c|c|c|}
\hline Tratamentos & & $\mathrm{pH}$ & $\mathbf{P}$ & K & $\mathrm{Ca}$ & Mg & $\mathrm{H}+\mathrm{Al}$ & $T$ & V\% \\
\hline Testemunha & & 3,8 & 5 & 1,3 & 1,4 & 1,0 & 5,0 & 8,8 & 41,0 \\
\hline Yoo pó & 50 ppm & 3,7 & 6 & 0,6 & 1,3 & 0,2 & 4,9 & 7,0 & 31,0 \\
\hline Yoo pó & 100 ppm & 3,7 & 9 & 0,6 & 1,5 & 0,1 & 5,2 & 7,5 & 29,0 \\
\hline Yoo pó & 200 ppm & 3,9 & 19 & 0,5 & 2,2 & 0,1 & 4,2 & 7,0 & 40,0 \\
\hline Y.Gros. & $50 \mathrm{ppm}$ & 3,5 & 9 & 0,6 & 1,3 & 0,2 & 5,4 & 7,5 & 29,0 \\
\hline Y.Gros. & $100 \mathrm{ppm}$ & 3,6 & 10 & 0,4 & 1,4 & 0,1 & 5,0 & 7,9 & 29,0 \\
\hline Y.Gros. & 200 ppm & 4,0 & 27 & 0,4 & 3,1 & 0,3 & 4,2 & 8,0 & 48,0 \\
\hline Y.Gran. & $50 \mathrm{ppm}$ & 3,7 & 5 & 0,5 & 1,4 & 0,4 & 4,9 & 7,2 & 32,0 \\
\hline Y.Gran. & $100 \mathrm{ppm}$ & 3,7 & 8 & 0,3 & 1,4 & 0,2 & 5,3 & 7,2 & 28,0 \\
\hline Y.Gran. & 200 ppm & 3,9 & 29 & 0,5 & 3,1 & 0,4 & 3,9 & 7,9 & 47,0 \\
\hline SPS & $50 \mathrm{ppm}$ & 3,6 & 10 & 0,5 & 1,1 & 0,1 & 5,8 & 7,6 & 23,0 \\
\hline SPS & $100 \mathrm{ppm}$ & 3,7 & 21 & 0,6 & 1,3 & 0,1 & 5,0 & 7,0 & 27,0 \\
\hline SPS & $200 \mathrm{ppm}$ & 3,6 & 43 & 0,5 & 2,0 & 0,1 & 6,2 & 8,4 & 29,0 \\
\hline
\end{tabular}

$\mathrm{P}\left(\mathrm{ug} / \mathrm{cm}^{3}\right) ; \mathrm{K}$ (e.mg/100 ml); Ca e Mg (e.mg/100 ml);H + Al (e.mg/100 ml); T (e.mg/100 ml).YPO-Yoorin Pó; YGR-Yoorin Grosso; YGN-Yoorin Granulado; SPS-superfosfato simples.

TABELA 6- $\mathbf{P}$ absorvido e recuperado para as diferentes fontes.

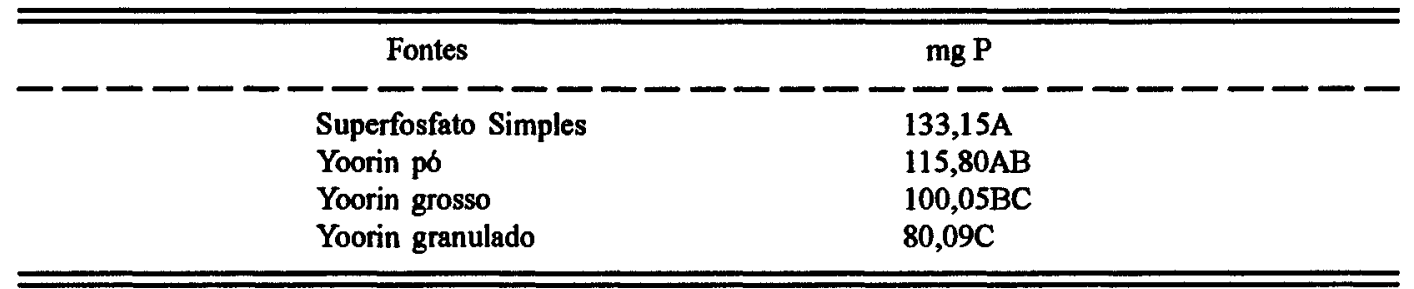

Médias seguidas de letras maiúsculas iguais, nas colunas, não diferem entre si a $1 \%$.

sas fontes, destacando o efeito da granulometria (TABELA 6).

O fósforo extraído e acumulado nos sete cultivos apresentou resultados estatisticamente agrupados, com um grupo intermediário cujas médias não diferiram entre si. Ficou clara a maior disponibilidade do fósforo para as fontes SPS e Yoorin pó, que não diferiram entre si a $1 \%$ de probabilidade. A inferioridade do termofosfato granulado é confirmada através da TABELA 6 , diferindo a $1 \%$ do primeiro grupo. O termofosfato de granulometria grosseira teve um comportamento intermediário aos de granulometrias pó e granulada, somente diferindo da fonte solúvel. Os resultados mostraram que a variação da granulometria nos termofosfatos interferiu na liberação de fósforo no solo e no efeito residual.

Os resultados superaram os valores obtidos por TANAKA (1990) e SANDER \& EGHBALL (1988) na recuperação de fósforo do solo pelas plantas. VASCONCELLOS et al. (1986); GOEDERT \&
LOBATO (1984); MURAOKA \& NEPTUNE (1978) encontraram maiores efeitos residuais para $o$ termofosfato que para fertilizantes solúveis, o que difere em parte dos resultados obtidos.

\section{CONCLUSÕES}

Nas condiçð̃es em que foram conduzidos os ensaios, os resultados permitem tirar as seguintes conclusões:

- O uso do termofosfato na forma pó, apresenta efeito residual semelhante ao do superfosfato simples.

- A granulometria mais grosseira do produto termofosfato resultou menor aproveitamento do fósforo residual.

\section{REFERENCIAS BIBLIOGRÁFICAS}

GOEDERT, W.J.; LOBATO, E. Avaliação agronômica de fosfatos em solo de cerrado. Revista Brasileira de Clêncla do Solo, Campinas, v.8, p.97-102, 1984. 
MALAVOLTA, E. Manual de quimica agricola: adubos e adubaçå̃o. 3.ed. São Paulo: Agronômica Ceres, 1981. 596p.

MALAVOLTA, E.; KLIEMANN, H.J. Desordens nutriclonais no cerrado. Piracicaba: POTAFÓS, 1985. 136p.

MALAVOLTA, E; VITTI, G.C.; OLIVEIRA, S.A. Avallafăo do estado nutriclonal das plantas: principios e aplicaç̃es. Piracicaba: POTAFós, 1989. 201p.

MURAOKA, T.; NEPTUNE, A.M.L. Eficiência de vários adubos fosfatados. II. Efeito residual. Anais da Escola Superior de Agricultura "Luiz de Queiroz", Piracicaba, v.35, p.533-539, 1978.

NOVOA, F.V.; NUNEZ, R. Efficiency of five phosphate fertilizer sources in soils with different phosphate fixing capacities. Tropical Agriculture, Guildford, v.2, p.235245, 1974.

RAIJ, B. van; ROSAND, P.C.; LOBATO, E. Adubaçăo fosfatada no Brasil: apreciação geral, conclusōes e recomendaçð̃es. In: OLIVEIRA, A.J.; LOURENÇO, S.; GOEDERT, W.J. (Eds.) Adubação fosfatada no Brasil. Brasilia: EMBRAPA/DID, 1982. p.9-28.

SANDER, D.H.; EGHBALL, B. Effect of fertilizer phosphurus particle size on phosphorus fertilizer eficiency. Joumal of Soll Sclence Society of America, Madison, v.52, p.868-873, 1988.
STEFANUTTI, R; MALAVOLTA, E.; MURAOKA, T. Comportamento de quatro extratores para fósforo no solo, com aplicacão de doses crescentes e diferentes fontes de P. IN: REUNIÃO BRASILEIRA DE FERTILIDADE DO SOLO E NUTRIÇAO DE PLANTAS, 20., Piracicaba, 1992. Anals... Piracicaba: SBCS, 1992. p.66-67.

TANAKA, R.T. Efeito do método de aplicação e do periodo de incubação na eficiência agronômica dos fosfatos. Piracicaba, 1990. 122p. Tese (Doutorado) - Escola Superior de Agricultura "Luiz de Queiroz", Universidade de Sao Paulo.

TERMAN, G.L.; ALLEN, S.E.; ENGELSTAD, O.P. Response by paddy rice to rates and sources applied phosphorus. Agronomy Journal, Madison, v.62, p.390$394,1970$.

VASCONCELLOS, C.A.; SANTOS, H.L.; FRANÇA, G.E. de; PITTA, G.V.E.; BAHIA FILHO, A.F.C. Eficiência agronômica de fosfatos naturais para a cultura do sorgo granifero: 1. Fósforo total e solúvel em ácido cítrico e granulometria. Revista Brasileira de Clência do Solo, Campinas, v.10, p.117-121, 1986.

VOLKWEISS, S.J.; RAIJ, B. van. Retenção e disponibilidade de fósforo em solos. In: SIMPÓSIO SOBRE CERRADO, 4., 1976, Brasilia. Bases para utilizaçăo agropecuária. Anais... Sæo Paulo: EDUSP, 1977. p.317-332.

Recebido para publicą̧ão em 26.08 .94 Aceito para publicação em 04.05 .95 